\title{
Multi-Objective Probabilistic Fractional Programming Problem Involving Two Parameters Cauchy Distribution
}

\author{
Srikumar Acharya, Berhanu Belay and Rajashree Mishra \\ Department of Mathematics, School of Applied Sciences, KIIT Deemed to be \\ University \\ Bhubaneswar, 751024, India \\ E-mail: rajashreemishra011@gmail.com, rmishrafma@kiit.ac.in \\ E-mail(corresp.): 1681121@kiit.ac.in, berhanubelay2@gmail.com \\ E-mail: acharyafma@kiit.ac.in, srikumar.kgp@gmail.com
}

Received June 6, 2018; revised April 15, 2019; accepted April 17, 2019

\begin{abstract}
The paper presents the solution methodology of a multi-objective probabilistic fractional programming problem, where the parameters of the right hand side constraints follow Cauchy distribution. The proposed mathematical model can not be solved directly. The solution procedure is completed in three steps. In first step, multi-objective probabilistic fractional programming problem is converted to deterministic multi-objective fractional mathematical programming problem. In the second step, it is converted to its equivalent multi-objective mathematical programming problem. Finally, $\epsilon$-constraint method is applied to find the best compromise solution. A numerical example and application are presented to demonstrate the procedure of proposed mathematical model.
\end{abstract}

Keywords: multi-objective programming problem, probabilistic programming problem, fractional programming problem, $\epsilon$-constraint method.

AMS Subject Classification: 90C15; 90C29; 90C32.

\section{Introduction}

Linear Fractional Programming (LFP) problem is a mathematical programming problem where the objective function is the ratio of two linear functions subject to the constraints with linear equalities or inequalities. The Hungarian mathematicians, Martos and Whinston [14] developed linear fractional programming problem in 1960s. LFP problem is applied when the constraints and

Copyright (C) 2019 The Author(s). Published by VGTU Press

This is an Open Access article distributed under the terms of the Creative Commons Attribution License (http://creativecommons.org/licenses/by/4.0/), which permits unrestricted use, distribution, and reproduction in any medium, provided the original author and source are credited. 
objective functions are deterministic in nature. But many real world decision making problems have uncertain objective functions and constraints due to incomplete or vague or uncertain information. Such type of uncertainties are addressed via fuzzy programming problem or stochastic programming problem. The mathematical models are said to be stochastic linear fractional programming problem, if some or all parameters of linear fractional programming are considered as random variables. There are two methods to deal with stochastic programming problem, namely two stage programming problem and chance constrained (probabilistic) programming problem.

Optimizing two or more fractional objective functions which are conflicting in nature is called multi-objective fractional programming problem. Multiobjective fractional programming problem is a beneficial means of modeling real life decision making problems with objective functions in the form of fractions such as minimizing actual cost/standard cost, maximizing profit/cost, minimizing inventory/sales, etc. Multi-objective LFP problem is also applicable in various disciplines like production planning problem, planning of agricultural problem, inventory problem, health care and hospital planning, financial planning, etc.

Multi-objective probabilistic linear fractional programming (MOPLFP) problem is nothing but it is optimizing the multi-objective linear fractional functions subject to some constraints which follows known random distribution. MOPLFP problem can not be solved directly. In order to solve it, the proposed model is transformed to known standard mathematical model in three steps. In the first step of transformation, MOPLFP problem is converted to its equivalent deterministic multi-objective linear fractional mathematical programming problem. In second step, multi-objective fractional mathematical programming problem is transformed to its equivalent multi-objective linear programming problem. Finally, multi-objective mathematical programming problem is solved using $\epsilon$-constrained method.

This paper has been organized into nine sections. The Section 1 presents the brief introduction of fractional programming problem. Section 2 presents motivation of the study. Section 3 presents review of literatures that are related to proposed work. Section 4 states the general model of MOPLFP problem. Section 5 presents the procedure to obtain deterministic equivalence of probabilistic programming problem. Section 6 presents the conversion of multiobjective linear fractional programming into equivalent multi-objective linear programming problem. Section 7 presents the solution procedure of multiobjective probabilistic linear fractional programming problem. One numerical and one practical example are given in Section 8 to illustrate the procedure. Finally, conclusions and references are provided in Section 9.

\section{Motivation}

Uncertain programming problem is an integral part of real life optimization problems. In most of the situations decision makers solve optimization problems that involves specific parameters which are mostly unknown. This leads to uncertainties in the input model parameters. Therefore parameters are han- 
dled by using known probability distribution functions. Stochastic programming is formulated due to presence of randomness in optimization problems. Most real life mathematical models involve more than one objective function which are conflicting in nature. Unlike single objective programming problems which give unique optimal solution, there may be many compromise solutions in multi-objective programming problem. Solving multi-objective programming problem is not an easy task. Sometimes decision makers need to determine the allocation (ratio) of resources to achieve certain specification that maximize or minimize the ratios. This motivates us to conduct and consider the proposed mathematical model as multi-objective probabilistic fractional programming problem.

\section{$3 \quad$ Literature survey}

To solve fractional programming problem, researchers presented the transformation of fractional programming problem into its equivalent mathematical programming problem. Chakraborty and Gupta [3] presented fuzzy programming method to obtain solution of multi-objective fractional mathematical programming problem after converting multi-objective fractional programming to multi-objective linear mathematical programming problem. An iterative parametric method is suggested by Valipour et al. [26] to obtain the solution of multi-objective linear fractional mathematical programming problem. A computer based method is developed by Hasan and Acharjee [1] to solve single objective linear fractional mathematical programming after converting into equivalent linear mathematical programming. Kornbluth and Steuer [12] presented a simplex-based method for the solution of multi-objective fractional mathematical programming problem. Latter Ponnaiah and Mohan [16] proposed a simplex method to find the solution of linear fractional mathematical programming problems by restricting the denominator of objective function. A fortran computer programme is developed by Saha et al. [20] to find the solution of LFP by transforming to a linear programming problem where the constant term of the denominator and numerator are negative. Odior [15] adopted a solution method for linear fractional mathematical programming problems using the concept of duality and partial fractions. Taylor series method was proposed by Güzel and Sivri [11] to find the solution of multi-objective linear fractional programming problem. Tantawy [24] developed iterative technique to find solution of linear fractional mathematical programming problems based on conjugate gradient projection. A method to convert stochastic sum of probabilistic fractional program problems in to stochastic constraints is presented by Charles and Dutta [6]. Wen and Wu [27] proposed a parametric method to solve linear fractional mathematical programming problems. Gradient method presented by Enkhbat et al. [10] to find the solution of convex concave fractional minimization problems. Roy [19] proposed genetic algorithm for solving single objective fractional programming problems. It is possible to convert linear fractional mathematical programming to equivalent linear mathematical programming by adding one extra [9]constraint and variable. Marchi [13] extended the transformation of Charnes and Cooper to other problems, where 
the functional involves quotient of linear functions. As Borza et al. [2] presented, the methodology for solution of LFP problems having interval coefficients in the objective function by using variable transformation. Bibliography on fractional mathematical programming has been presented by Stancu $[22,23]$. Udhayakumar et al. [25] solved probabilistic fractional programming problems using stochastic simulation based genetic algorithm, where random variables follow any continuous distribution. Charles and Dutta [5] obtained the efficient solution of multi-objective stochastic fractional mathematical programming problems when the continuous random variables involved in the objective functions and constraints. Charles and Dutta [8] presented a method to recognize redundant objective functions for a given multi-objective probabilistic fractional mathematical programming.

Some applications of linear fractional mathematical programming problems are studied by many researchers. Charles and Dutta [4] applied linear probabilistic fractional mathematical programming by using branch and bounded method for machine manufacturing problem. Ren et al. [17] developed a model for the uncertainty of water resources allocation for industry having probabilistic mathematical programming problem and fractional programming problem. Ren et al. [18] developed a model for optimal allocation of uncertain water resources for industries by mixing probabilistic mathematical programming and fractional programming. Charles and Dutta [7] presented application of multiobjective stochastic fractional programming for assembled printed circuit board problem. Zhu and Huang [28] presented the application of probabilistic fractional programming problem for maintaining municipal solid waste controlling under uncertainty. Latter Zhu and Huang [29] presented dynamic stochastic fractional programming approach for sustainable management of electric power under uncertainty.

In this paper, we study MOPLFP problem where parameters in the right hand side of the constraints follow Cauchy distribution. To solve the problem, we convert the fractional mathematical programming problem having multiobjective functions into equivalent multi-objective mathematical programming problem and probabilistic programming problem into equivalent deterministic programming problem. Finally, $\epsilon$-constraint technique is used to obtain the solution.

\section{Formulation of multi-objective probabilistic linear fractional programming problem}

A MOPLFP problem is expressed as:

$$
\max : Z_{q}=\frac{N_{q}\left(x_{j}\right)}{D_{q}\left(x_{j}\right)}=\frac{\sum_{j=1}^{n} c_{q j} x_{j}+\alpha_{q}}{\sum_{j=1}^{n} d_{q j} x_{j}+\beta_{q}}, q=1,2, \ldots, Q
$$

subject to

$$
P\left(\sum_{j=1}^{n} a_{i j} x_{j} \leq b_{i}\right) \geq \eta_{i}, i=1,2, \ldots, m
$$




$$
0<\eta_{i}<1, \quad i=1,2, \ldots, m, \quad x_{j} \geq 0, \quad j=1,2, \ldots, n,
$$

where

$$
N_{q}\left(x_{j}\right)=\sum_{j=1}^{n} c_{q j} x_{j}+\alpha_{q}, \quad D_{q}\left(x_{j}\right)=\sum_{j=1}^{n} d_{q j} x_{j}+\beta_{q} .
$$

The functions $N_{q}\left(x_{j}\right)$ and $D_{q}\left(x_{j}\right)$ are linear functions of $x_{j}, c_{q j}, d_{q j} \in R^{n}$, $a_{i j} \in R^{m \times n}, \alpha_{q}$ and $\beta_{q}$ are scalars, "P" indicates probability, $\eta_{i}$ is the given probability at which the $i^{\text {th }}$ constraint violations are admitted.

\section{Deterministic equivalence of probabilistic constraints}

We assumed that the random variables $b_{i}$ follow Cauchy distribution with two parameters $\gamma_{i}$ and $\delta_{i}$. The parameters $\delta_{i}$ and $\gamma_{i}$ denote locations and scales of the random variables $b_{i}$. The probability density function (pdf) of Cauchy random variable $b_{i}$ is expressed by:

$$
f\left(b_{i}\right)=\frac{\gamma_{i}}{\pi\left(\gamma_{i}^{2}+\left(b_{i}-\delta_{i}\right)^{2}\right)}, \quad-\infty<b_{i}<\infty, \quad-\infty<\delta_{i}<\infty, \gamma_{i}>0 .
$$

There are different methods of estimating parameters of Cauchy distribution. To select the location and scale parameters of random sample of Cauchy distribution, one can use maximum likelihood estimation.

The procedure of selecting parameters of Cauchy distribution is described as follows. Let $x_{1}, x_{2}, \ldots, x_{n}$ be random sample of Cauchy distribution. The likelihood function $L\left(\delta, \gamma \mid x_{1}, x_{2}, \ldots, x_{n}\right)$ is a function that selects the value of the parameter values $\delta$ and $\gamma$ where the data is most probable. Maximum likelihood is a technique that helps to find the value of the parameters that maximize the likelihood function. The log-likelihood function of the Cauchy distribution is given by:

$$
\hat{L}\left(\delta, \gamma \mid x_{1}, x_{2}, \ldots, x_{n}\right)=-\log (\pi \gamma)-\sum_{i=1}^{n} \log \left(1+\frac{\left(x_{i}-\delta\right)^{2}}{\gamma^{2}}\right) .
$$

Putting $\frac{\partial \hat{L}}{\partial \delta}=0$ and $\frac{\partial \hat{L}}{\partial \gamma}=0$, it is possible to find the maximum value of the likelihood function.

$$
\begin{aligned}
& \frac{\partial \hat{L}}{\partial \delta}\left(\delta, \gamma \mid x_{1}, x_{2}, \ldots, x_{n}\right)=\sum_{i=1}^{n} \frac{x_{i}-\delta}{\left(x_{i}-\delta\right)^{2}+\gamma^{2}}=0 \\
& \frac{\partial \hat{L}}{\partial \gamma}\left(\delta, \gamma \mid x_{1}, x_{2}, \ldots, x_{n}\right)=\sum_{i=1}^{n} \frac{\gamma^{2}}{\left(x_{i}-\delta\right)^{2}+\gamma^{2}}=\frac{n}{2}
\end{aligned}
$$

Solving (5.1) and (5.2) simultaneously, one can find the values of the two parameters $\gamma$ and $\delta$. From the probabilistic constraint (4.2), we have

$$
P\left(\sum_{j=1}^{n} a_{i j} x_{j} \leq b_{i}\right) \geq \eta_{i}, \quad \Rightarrow \quad P\left(b_{i} \geq \sum_{j=1}^{n} a_{i j} x_{j}\right) \geq \eta_{i} .
$$


Let $y_{i}=\sum_{j=1}^{n} a_{i j} x_{j}$. Then the probabilistic constraint (5.3) is written as

$$
\int_{y_{i}}^{\infty} \frac{\gamma_{i}}{\pi\left(\gamma_{i}^{2}+\left(b_{i}-\delta_{i}\right)^{2}\right)} d b_{i} \geq \eta_{i}, i=1,2, \ldots, m
$$

After integration, we have

$$
\frac{1}{\pi}\left[\tan ^{-1}\left(\frac{b_{i}-\delta_{i}}{\gamma_{i}}\right)\right]_{y_{i}}^{\infty} \geq \eta_{i}
$$

Substituting the limit of integration, we obtain the following result

$$
\frac{\pi}{2}-\tan ^{-1} \frac{y_{i}-\delta_{i}}{\gamma_{i}} \geq \eta_{i} \pi
$$

which is simplified as

$$
\tan ^{-1} \frac{y_{i}-\delta_{i}}{\gamma_{i}} \leq \frac{\pi}{2}-\eta_{i} \pi
$$

Taking tangent both sides of (5.4), we obtain

$$
\frac{y_{i}-\delta_{i}}{\gamma_{i}} \leq \tan \left(\frac{\pi}{2}-\eta_{i} \pi\right)
$$

solving for $y_{i}$, we have

$$
y_{i} \leq \delta_{i}+\gamma_{i} \tan \left(\frac{\pi}{2}-\eta_{i} \pi\right) \Rightarrow \sum_{j=1}^{n} a_{i j} x_{j} \leq \delta_{i}+\gamma_{i} \tan \left(\frac{\pi}{2}-\eta_{i} \pi\right) .
$$

Substituting (5.5) in (4.2), the deterministic equivalent of the MOPLFP problem (4.1)-(4.3) is expressed as follows:

$$
\max : Z_{q}=\frac{N_{q}\left(x_{j}\right)}{D_{q}\left(x_{j}\right)}, q=1,2, \ldots, Q
$$

subject to

$$
\begin{aligned}
& \sum_{j=1}^{n} a_{i j} x_{j} \leq \delta_{i}+\gamma_{i} \tan \left(\frac{\pi}{2}-\eta_{i} \pi\right), i=1,2, \ldots, m, \\
& 0<\eta_{i}<1, i=1,2, \ldots, m, \quad x_{j} \geq 0, j=1,2, \ldots, n .
\end{aligned}
$$

\section{Conversion of multi-objective linear fractional programming problem into equivalent multi-objective linear programming problem}

Consider a single objective linear fractional mathematical programming problem

$$
\max : Z_{1}=\frac{c_{1 j} x_{j}+\alpha}{d_{1 j} x_{j}+\beta}
$$


subject to

$$
\sum_{j=1}^{n} a_{i j} x_{j} \leq b_{i}, i=1,2, \ldots, m, \quad x_{j} \geq 0, c_{1 j}, d_{1 j} \in R^{n}, \quad \alpha, \beta \in R .
$$

For simplicity, assume that $d_{1 j} x_{j}+\beta>0, x_{j} \geq 0$ and $\sum_{j=1}^{n} a_{i j} x_{j} \leq b_{i}$. Using the transformation

$$
y_{j}=x_{j} t, \quad t=\frac{1}{d_{1 j} x_{j}+\beta}
$$

then $(6.1)-(6.2)$ is equivalent to

$$
\max : Z_{1}=c_{1 j} y_{j}+\alpha t
$$

subject to

$$
\begin{aligned}
& d_{1 j} y_{j}+\beta t=1, \quad \sum_{j=1}^{n} a_{i j} y_{j}-t b_{i} \leq 0, i=1,2, \ldots, m, \\
& t>0, \quad y_{j} \geq 0, \quad t \in R .
\end{aligned}
$$

Consider the concave-convex programming problem

$$
\max : Z_{1}=\frac{N_{1}\left(x_{j}\right)}{D_{1}\left(x_{j}\right)}
$$

subject to

$$
\sum_{j=1}^{n} a_{i j} x_{j} \leq b_{i}, i=1,2, \ldots, m, \quad D_{1}\left(x_{j}\right)>0, x_{j} \geq 0 .
$$

Using the transformation $y_{j}=x_{j} t$ and $t=\frac{1}{D_{1}\left(x_{j}\right)}$, the programming problem (6.3)-(6.4) is written as

$$
\max : Z_{1}=t N_{1}\left(y_{j} / t\right)
$$

subject to

$$
\sum_{j=1}^{n} a_{i j}\left(y_{j} / t\right) \leq b_{i}, i=1,2, \ldots, m, \quad t D_{1}\left(y_{j} / t\right)=1, \quad y_{j} \geq 0, t>0 .
$$

Definition 1. Equation (6.3)-(6.4) is called concave-convex fractional programming if the following conditions are satisfied:

i) $N_{1}\left(x_{j}\right)$ is concave function and $N_{1}\left(x_{j}\right)>0$, for some $x_{j}$ in the feasible space.

ii) $D_{1}\left(x_{j}\right)$ is convex function and positive.

Theorem 1 [Schaible [21]]. If (6.3)-(6.4) has global maximum at $x_{j}=x_{j}^{*}$, then (6.5)-(6.6) has global maximum at $\left(t, y_{j}\right)=\left(t^{*}, y_{j}^{*}\right), x_{j}^{*}=y_{j}^{*} / t^{*}$. 
Theorem 2 [Schaible [21]]. If (6.3)-(6.4) is concave-convex LFP problem having global maximum at point $x^{*}$, then (6.5)-(6.6) attains maximum value at the point $\left(t^{*}, y_{j}^{*}\right), x_{j}^{*}=y_{j}^{*} / t^{*}$.

But if $N_{1}\left(x_{j}\right)$ is concave and negative and $D_{1}\left(x_{j}\right)$ concave and positive, then $-N_{1}\left(x_{j}\right)$ is convex and positive. Hence (6.3)-(6.4) is changed to the following linear mathematical programming problem

$$
\max : t D_{1}\left(y_{j} / t\right)
$$

subject to

$$
\sum_{j=1}^{n} a_{i j}\left(y_{j} / t\right) \leq b_{i}, i=1,2, \ldots, m, \quad-t N_{1}\left(y_{j} / t\right)=1, \quad y_{j} \geq 0, t>0 .
$$

Now, let us extend the single objective LFP problem into multi-objective fractional programming problem which is expressed in Section 5 by:

$$
\begin{aligned}
& \max : Z_{q}=\frac{N_{q}\left(x_{j}\right)}{D_{q}\left(x_{j}\right)}, q=1,2, \ldots Q, \\
& \sum_{j=1}^{n} a_{i j} x_{j} \leq \delta_{i}+\gamma_{i} \tan \left(\frac{\pi}{2}-\eta_{i} \pi\right), i=1,2, \ldots, m, \\
& 0<\eta_{i}<1, i=1,2, \ldots, m, \quad x_{j} \geq 0, \quad j=1,2, \ldots, n .
\end{aligned}
$$

For some $x_{j}$ in the feasible set, consider the two index sets

$$
I=\left\{q: N_{q}\left(x_{j}\right) \geq 0\right\}, \quad I^{c}=\left\{q: N_{q}\left(x_{j}\right)<0\right\},
$$

where

$$
I \bigcup I^{c}=\{1,2, \ldots, Q\} .
$$

Let $D_{q}\left(x_{j}\right)$ be positive on the feasible set. For simplicity let us take

$$
\bigcap_{q \in I} \frac{1}{d_{q j} x_{j}+\beta_{i}}=t, \quad \bigcap_{q \in I^{c}} \frac{-1}{c_{q j} x_{j}+\alpha_{i}}=t .
$$

This is equivalent to

$$
\frac{1}{d_{q j} x_{j}+\beta_{i}} \geq t, q \in I, \quad \frac{-1}{c_{q j} x_{j}+\alpha_{i}} \geq t, q \in I^{c} .
$$

Using the transformation $y_{j}=t x_{j}$, the equivalent multi-objective linear programming problem for (6.7)-(6.8) is given by:

$$
\max : Z_{q}=t N_{q}\left(y_{j} / t\right) \text {, if } \mathrm{q} \in I, t D_{q}\left(y_{j} / t\right) \text {, if } \mathrm{q} \in I^{c}, q=1,2, \ldots, Q
$$

subject to

$$
\begin{aligned}
& \sum_{j=1}^{n} a_{i j}\left(y_{j} / t\right)-\left(\delta_{i}+\gamma_{i} \tan \left(\pi / 2-\eta_{i} \pi\right)\right) \leq 0, i=1,2, \ldots, m, \\
& t D_{q}\left(y_{j} / t\right)=1, \quad \text { if } \mathrm{q} \in I, \quad-t N_{q}\left(y_{j} / t\right)=1, \quad \text { if } \mathrm{q} \in I^{c}, \\
& 0<\eta_{i}<1, \quad i=1,2, \ldots, m, \quad t>0, y_{j} \geq 0, j=1,2, \ldots, n,
\end{aligned}
$$

where the constraint set is convex set having feasible points. 


\section{Solution procedure}

It is difficult to solve the mathematical programming problem (4.1)-(4.3) directly. In this paper, we use $\epsilon$-constraint approach after the problem is changed to deterministic multi-objective programming problem. The basic steps of $\epsilon$-constraint method for multi-objective linear programming problem are described as follows.

Step 1: Find the first ideal solution $X^{(1)}$ by solving the first objective function disregarding the other objective functions.

$$
\max : Z_{1}=t N_{1}\left(y_{j} / t\right) \text {, if } 1 \in I, t D_{1}\left(y_{j} / t\right) \text {, if } 1 \in I^{c}
$$

subject to

$$
\begin{aligned}
& \sum_{j=1}^{n} a_{i j}\left(y_{j} / t\right)-\left(\delta_{i}+\gamma_{i} \tan \left(\pi / 2-\eta_{i} \pi\right)\right) \leq 0, i=1,2, \ldots, m, \\
& t D_{1}\left(y_{j} / t\right)=1, \quad \text { if } 1 \in I, \quad-t N_{1}\left(y_{j} / t\right)=1, \quad \text { if } 1 \in I^{c} \\
& 0<\eta_{i}<1, \quad i=1,2, \ldots, m, \quad t>0, \quad y_{j} \geq 0, \quad j=1,2, \ldots, n .
\end{aligned}
$$

Similarly, ideal solutions for second, third and so on are obtained by solving individual mathematical programming problem.

Step 2: With the help of ideal solutions found in Step 1, construct a pay-off matrix which is shown in Table 1. Estimate the upper and lower bounds of the objective functions from the pay-off matrix as $L_{q} \leq Z_{q} \leq U_{q}, q=1,2, \ldots, Q$.

Step 3: Determine the bounds of $\epsilon_{q}, q=1,2, \ldots, Q$ which is the point in the range of $Z_{q}$.

Step 4: Using $\epsilon_{q}(q=1,2, \ldots, Q)$, construct and solve $Q$ different single objective programming problem.

$i)$ : For $q=1$ optimize $Z_{1}(x)$ subject to the original constraints and new constraints $Z_{q}(x) \geq \epsilon_{q}, q=1,2, \ldots, Q(q \neq 1)$, i.e.,

$$
\max : Z_{1}=t N_{1}\left(y_{j} / t\right) \text {, if } 1 \in I, t D_{1}\left(y_{j} / t\right), \text { if } 1 \in I^{c}
$$

subject to

$$
\begin{aligned}
& \sum_{j=1}^{n} a_{i j}\left(y_{j} / t\right)-\left(\delta_{i}+\gamma_{i} \tan \left(\pi / 2-\eta_{i} \pi\right)\right) \leq 0, \quad i=1,2, \ldots, m, \\
& t N_{q}\left(y_{j} / t\right) \leq \epsilon_{q}, \quad \text { if } \mathrm{q} \in I, q \neq 1, \quad t D_{q}\left(y_{j} / t\right) \leq \epsilon_{q}, \quad \text { if } \mathrm{q} \in I^{c}, \quad q \neq 1, \\
& t D_{1}\left(y_{j} / t\right)=1, \quad \text { if } 1 \in I, \quad-t N_{1}\left(y_{j} / t\right)=1, \quad \text { if } 1 \in I^{c} \\
& 0<\eta_{i}<1, \quad i=1,2, \ldots, m, \quad t>0, \quad y_{j} \geq 0, \quad j=1,2, \ldots, n .
\end{aligned}
$$

ii) : For $q=2$ optimize $Z_{2}$ subject to the original constraints and new constraints $Z_{q}(x) \geq \epsilon_{q}, q=1,2, \ldots, Q(q \neq 2)$.

iii) : Proceed the procedure $Q$ times for $Q$ objective functions.

Step 5: Solve the single objective mathematical model by using suitable mathematical method or software to obtain optimal compromise solution for different values of $\epsilon_{q}, q=1,2, \ldots, Q$. 
Table 1. Pay-off matrix.

\begin{tabular}{rrrrr}
\hline & $Z_{1}(X)$ & $Z_{2}(X)$ & $\cdots$ & $Z_{Q}(X)$ \\
\hline$X^{(1)}$ & $Z_{1}\left(X^{(1)}\right)$ & $Z_{2}\left(X^{(1)}\right)$ & $\ldots$ & $Z_{Q}\left(X^{(1)}\right)$ \\
$X^{(2)}$ & $Z_{1}\left(X^{(2)}\right)$ & $Z_{2}\left(X^{(2)}\right)$ & $\cdots$ & $Z_{Q}\left(X^{(2)}\right)$ \\
$\vdots$ & $\vdots$ & $\vdots$ & & $\vdots$ \\
$X^{(Q)}$ & $Z_{1}\left(X^{(Q)}\right)$ & $Z_{2}\left(X^{(Q)}\right.$ & $\cdots$ & $Z_{Q}\left(X^{(Q)}\right.$ \\
\hline
\end{tabular}

\section{Examples}

In this section, we see one numerical example and one practical example on nurse scheduling problem to illustrate the proposed model.

\subsection{Numerical example}

Consider a MOPLFP problem where the parameters of the right hand side constraints follow Cauchy distribution.

$$
\min : Z_{1}=\frac{2 x_{1}+5 x_{2}+4 x_{3}+8}{3 x_{1}+6 x_{2}+5 x_{3}+2}, \quad \max : Z_{2}=\frac{5 x_{1}+4 x_{2}+6 x_{3}+7}{5 x_{1}+3 x_{2}+4 x_{3}+5}
$$

subject to

$$
\begin{aligned}
& P\left(7 x_{1}+2 x_{2}+4 x_{3} \leq b_{1}\right) \geq 0.90, \quad P\left(5 x_{1}+1 x_{2}+6 x_{3} \leq b_{2}\right) \geq 0.75 \\
& P\left(x_{1}+2 x_{2}+x_{3} \leq b_{3}\right) \geq 0.80, \quad P\left(x_{1}+x_{2}+2 x_{3} \leq b_{4}\right) \geq 0.95 \\
& x_{j} \geq 0, j=1,2,3
\end{aligned}
$$

where $b_{1}, b_{2}, b_{3}, b_{4}$ are random variables that have Cauchy distribution with two known parameters $\delta_{1}=200, \gamma_{1}=2, \delta_{2}=400, \gamma_{2}=4, \delta_{3}=100, \gamma_{3}=3$, $\delta_{4}=300, \gamma_{4}=6$. Now, first let us change the MOPLFP problem into it's deterministic equivalent multi-objective linear fractional mathematical programming problem using (5.6)-(5.7).

$$
\min : Z_{1}=\frac{2 x_{1}+5 x_{2}+4 x_{3}+8}{3 x_{1}+6 x_{2}+5 x_{3}+2}, \quad \max : Z_{2}=\frac{5 x_{1}+4 x_{2}+6 x_{3}+7}{5 x_{1}+3 x_{2}+4 x_{3}+5}
$$

subject to

$$
\begin{aligned}
& 7 x_{1}+2 x_{2}+4 x_{3} \leq \delta_{1}+\gamma_{1} \tan \left(\pi / 2-\eta_{1} \pi\right), \\
& 5 x_{1}+1 x_{2}+6 x_{3} \leq \delta_{2}+\gamma_{2} \tan \left(\pi / 2-\eta_{2} \pi\right), \\
& x_{1}+2 x_{2}+x_{3} \leq \delta_{3}+\gamma_{3} \tan \left(\pi / 2-\eta_{3} \pi\right), \\
& x_{1}+x_{2}+2 x_{3} \leq \delta_{4}+\gamma_{4} \tan \left(\pi / 2-\eta_{4} \pi\right), \quad x_{j} \geq 0, \quad j=1,2,3 .
\end{aligned}
$$

Substituting all the values of $\delta_{i}, \gamma_{i}, \eta_{i}, i=1,2,3,4$ and simplifying the above mathematical problem we obtain.

$$
\min : Z_{1}=\frac{2 x_{1}+5 x_{2}+4 x_{3}+8}{3 x_{1}+6 x_{2}+5 x_{3}+2}, \quad \max : Z_{2}=\frac{5 x_{1}+4 x_{2}+6 x_{3}+7}{5 x_{1}+3 x_{2}+4 x_{3}+5}
$$


subject to

$$
\begin{aligned}
& 7 x_{1}+2 x_{2}+4 x_{3} \leq 194, \quad 5 x_{1}+1 x_{2}+6 x_{3} \leq 396, \\
& x_{1}+2 x_{2}+x_{3} \leq 96, \quad x_{1}+x_{2}+2 x_{3} \leq 262, \quad x_{j} \geq 0, \quad j=1,2,3 .
\end{aligned}
$$

Using the transformation $y_{j}=x_{j} t, y_{j} \geq 0, j=1,2,3$ and $t>0$, the equivalent multi-objective linear mathematical programming is given by:

$$
\begin{aligned}
& \min : f_{1}=2 y_{1}+5 y_{2}+4 y_{3}+8 t, \\
& \max : f_{2}=5 y_{1}+4 y_{2}+6 y_{3}+7 t
\end{aligned}
$$

subject to

$$
\begin{aligned}
& 3 y_{1}+6 y_{2}+5 y_{3}+2 t=1, \quad 5 y_{1}+3 y_{2}+4 y_{3}+5 t=1 \\
& 7 y_{1}+2 y_{2}+4 y_{3}-194 t \leq 0, \quad 5 y_{1}+y_{2}+6 y_{3}-396 t \leq 0, \\
& y_{1}+2 y_{2}+y_{3}-96 t \leq 0, \quad y_{1}+y_{2}+2 y_{3}-262 t \leq 0, y_{j} \geq 0, j=1,2,3, t>0 .
\end{aligned}
$$

The above multi-objective linear programming problem is solved by using $\epsilon$ constraint method. Solving the two functions separately, the two ideal solutions are:

$$
\begin{aligned}
& y^{(1)}=\left(y_{1}, y_{2}, y_{3}, t\right)=(0.1361059,0.09664461,0,0.005907372), \\
& y^{(2)}=\left(y_{1}, y_{2}, y_{3}, t\right)=(0,0,0.1764706,0.05882353)
\end{aligned}
$$

where the value of $f_{1}=0.8026938$ and $f_{2}=1.470588$. To determine the bounds of $\epsilon$ for each objective function, we have to construct a pay-off matrix. Hence using these ideal solutions a pay-off matrix is constructed as in Table 2.

Table 2. Pay-off matrix to find bounds of $\epsilon$.

\begin{tabular}{rrr}
\hline & $f_{1}(Y)$ & $f_{2}(Y)$ \\
\hline$y^{(1)}$ & 0.8026938 & 1.108459544 \\
$y^{(2)}$ & 1.17647064 & 1.470588 \\
\hline
\end{tabular}

From the pay-off matrix given by Table 2, the lower and the upper bounds of the two objective functions are given by:

$$
0.8026938 \leq f_{1}(y) \leq 1.17647064, \quad 1.108459544 \leq f_{2}(y) \leq 1.470588 .
$$

Therefore the bounds of $\epsilon_{q}, q=1,2$ is the point in the range of the objective function $f_{q}(y)$, i.e.,

$$
0.8026938 \leq \epsilon_{1} \leq 1.17647064, \quad 1.108459544 \leq \epsilon_{2} \leq 1.470588
$$

Using $\epsilon_{q}(q=1,2)$, we define two different single objective programming problems by taking one objective function as constraint as follows:

The first single objective programming problem is given by considering the second objective function $f_{2}$ as constraint.

$$
\min : f_{1}=2 y_{1}+5 y_{2}+4 y_{3}+8 t
$$


subject to

$$
\begin{aligned}
& 5 y_{1}+4 y_{2}+6 y_{3}+7 t \geq \epsilon_{2}, \quad 3 y_{1}+6 y_{2}+5 y_{3}+2 t=1, \\
& 5 y_{1}+3 y_{2}+4 y_{3}+5 t=1, \quad 7 y_{1}+2 y_{2}+4 y_{3}-194 t \leq 0, \\
& 5 y_{1}+y_{2}+6 y_{3}-396 t \leq 0, \quad y_{1}+2 y_{2}+y_{3}-96 t \leq 0, \\
& y_{1}+y_{2}+2 y_{3}-262 t \leq 0, \quad y_{j} \geq 0, \quad j=1,2,3, t>0 .
\end{aligned}
$$

Solving (8.1)-(8.2) by taking different values of $\epsilon_{2}$ in the interval [1.108459544, $1.470588]$ by LINGO software and applying the transformation $x_{i}=y_{i} / t, i=$ $1,2,3$, the solution of the original programming problem is given by Table 3 . From Table 3, we obtained different compromise solutions for the given pro-

Table 3. Solution of $Z_{1}$ and $Z_{2}$ for different values of $\epsilon_{2}$.

\begin{tabular}{rrrrrr}
\hline$\epsilon_{2}$ & $x_{1}$ & $x_{2}$ & $x_{3}$ & $Z_{1}$ & $Z_{2}$ \\
\hline 1.17 & 19.98946 & 11.784188 & 7.626354 & 0.8044557 & 1.1700 \\
1.25 & 15.94074 & 5.711112 & 17.748151 & 0.8067461 & 1.25000 \\
1.35 & 3.727271 & 0.000000 & 10.454542 & 0.8750000 & 1.3500 \\
1.4 & 1.000000 & 0.000000 & 5.000005 & 1.000000 & 1.4000 \\
1.47 & 0.047394 & 0.0000000 & 3.009478 & 1.175000 & 1.4700 \\
\hline
\end{tabular}

gramming problem. The optimal value of $Z_{2}$ is similar to $\epsilon_{2}$. The decision maker can select best compromise solutions by selecting appropriate value of $\epsilon_{2}$. The second single objective programming problem is given by considering the first objective function $f_{1}$ as a constraint.

$$
\max : f_{2}=5 y_{1}+4 y_{2}+6 y_{3}+7 t
$$

subject to

$$
\begin{aligned}
& 2 y_{1}+5 y_{2}+4 y_{3}+8 t \leq \epsilon_{1}, \quad 3 y_{1}+6 y_{2}+5 y_{3}+2 t=1, \\
& 5 y_{1}+3 y_{2}+4 y_{3}+5 t=1, \quad 7 y_{1}+2 y_{2}+4 y_{3}-194 t \leq 0, \\
& 5 y_{1}+y_{2}+6 y_{3}-396 t \leq 0, \quad y_{1}+2 y_{2}+y_{3}-96 t \leq 0, \\
& y_{1}+y_{2}+2 y_{3}-262 t \leq 0, \quad y_{j} \geq 0, \quad j=1,2,3, t>0 .
\end{aligned}
$$

Solving (8.3)-(8.4) by taking different values of $\epsilon_{1}$ in the interval [0.8026938, $1.17647064]$ by LINGO software and applying the transformation $x_{i}=y_{i} / t, i=$ $1,2,3$, the solution of the original programming problem is given by Table 4 . From Table 4, we obtain set of compromise solutions which optimize the two conflicting objective functions. A decision maker can choose one optimal solution among the set compromise solutions by selecting the appropriate value of $\epsilon_{1}$.

\subsection{Application on nurses schedule problem}

Nurses scheduling problem helps for any health organization to have sufficient number of nurses in each shift. In this example, we focus on nurse scheduling problem where the nurses manager wants to create a schedule for two shifts 
Table 4. Solution of $Z_{1}$ and $Z_{2}$ for different values of $\epsilon_{1}$

\begin{tabular}{rrrrrr}
\hline$\epsilon_{1}$ & $x_{1}$ & $x_{2}$ & $x_{3}$ & $Z_{1}$ & $Z_{2}$ \\
\hline 0.8 & 16.0000001 & 0.000000 & 35.000045 & 0.800 & 1.320000 \\
0.9 & 2.764705 & 0.000000 & 8.529411 & 0.900 & 1.360000 \\
1.0 & 1.000000 & 0.0000000 & 5.0000006 & 1.000 & 1.400000 \\
1.1 & 0.302325 & 0.0000000 & 3.604650 & 1.100 & 1.440000 \\
1.176 & 0.001513 & 0.0000000 & 3.003024 & 1.1720 & 1.470400 \\
\hline
\end{tabular}

namely day and night shifts for 1 week. The schedule is done by optimizing two objectives. The primary objective of any health organization is minimizing the total cost assigned for nurses. In this problem, the manager wants to minimize the the ratio of total over time cost and regular time cost assigned for nurses at each shift. The second objective is maximizing the ratio of senior nurses and junior nurses in each shift. The total number of over time hours assigned for each nurse in each shit must satisfy the maximum stay period of patients in the health care at each shift. Since the stay period of patients in the health care are unknown at each shift, the maximum stay period of patients in the health care is uncertain which is expressed by random variable following Cauchy distribution having location and scale parameters. The mathematical programming problem is expressed by:

$$
\begin{aligned}
& \min : Z_{1}=\frac{\sum_{n=1}^{2} \sum_{s=1}^{2} \sum_{t=1}^{7} o_{n} h_{s t} x_{n s t}}{\sum_{n=1}^{2} \sum_{s=1}^{2} \sum_{t=1}^{7} r_{n} x_{n s t}}, \quad \max : Z_{2}=\frac{\sum_{s=1}^{2} \sum_{t=1}^{7} x_{1 s t}}{\sum_{s=1}^{2} \sum_{t=1}^{7} x_{2 s t}}, \\
& P\left(\sum_{n=1}^{2} h_{s t} x_{n s t} \geq M_{s t}\right) \geq \eta_{s t}, \quad x_{n s t}>0,
\end{aligned}
$$

where $n$ is index of nurse in health care, $n=1$ represents senior nurse and $n=2$ represents junior nurse. $s=1,2$ is index of shift, $t=1,2, \ldots, 7$ is index of date in a week, $x_{n s t}$ is number of nurses needed in each shift on each day. $o_{n s}$ is over time cost assigned for each nurse at each shift, $r_{n s}$ is regular time cost assigned for each nurse at each shift, $h_{n s t}$ are over time hours assigned for nurse $n$ for each shift and date. $M_{s t}$ is the maximum period of patients staying in the health care at each shift in each time, $\eta_{s t}$ is the probability at which the constraint violations are admitted.

Assume that the total over time cost for senior and junior nurses is $\$ 30$ and $\$ 20$ per hour respectively, where as the total over time needed at each shift and date is given by Table 5 .

Table 5. Over time values in hours.

\begin{tabular}{rrrrrrrrrrrrrr}
\hline$h_{11}$ & $h_{21}$ & $h_{12}$ & $h_{22}$ & $h_{13}$ & $h_{23}$ & $h_{14}$ & $h_{24}$ & $h_{15}$ & $h_{25}$ & $h_{16}$ & $h_{26}$ & $h_{17}$ & $h_{27}$ \\
\hline 4 & 6 & 3 & 4 & 2 & 2 & 3 & 4 & 4 & 3 & 5 & 6 & 4 & 5 \\
\hline
\end{tabular}

Since the maximum over time in each shift and date is random variable following Cauchy distribution, the values of the two parameters $\delta$ and $\gamma$ are given 
by Table 6 . The probabilities at which the constraint violations admitted are

Table 6. Random variable parameter values.

\begin{tabular}{rrrrrrrrrrrrrrr}
\hline & $M_{11}$ & $M_{21}$ & $M_{12}$ & $M_{22}$ & $M_{13}$ & $M_{23}$ & $M_{14}$ & $M_{24}$ & $M_{15}$ & $M_{25}$ & $M_{16}$ & $M_{26}$ & $M_{17}$ & $M_{27}$ \\
\hline$\delta$ & 80 & 60 & 90 & 75 & 65 & 70 & 85 & 95 & 70 & 100 & 80 & 50 & 60 & 70 \\
$\gamma$ & 4 & 2 & 3 & 4 & 5 & 1 & 3 & 6 & 4 & 2 & 6 & 3 & 4 & 5 \\
\hline
\end{tabular}

given by Table 7. The regular time cost assigned for senior and junior nurses is

Table 7. Values of probabilities.

\begin{tabular}{rrrrrrrrrrrrrr}
\hline$\eta_{11}$ & $\eta_{21}$ & $\eta_{12}$ & $\eta_{22}$ & $\eta_{13}$ & $\eta_{23}$ & $\eta_{14}$ & $\eta_{24}$ & $\eta_{15}$ & $\eta_{25}$ & $\eta_{16}$ & $\eta_{26}$ & $\eta_{17}$ & $\eta_{27}$ \\
\hline 0.95 & 0.7 & 0.75 & 0.6 & 0.5 & 0.45 & 0.65 & 0.35 & 0.9 & 0.7 & 0.55 & 0.5 & 0.65 & 0.85 \\
\hline
\end{tabular}

$\$ 185$ and $\$ 120$ per day respectively. The total regular time per day is 8 hours. Since the formulated nurses scheduling problem is multi-objective probabilistic fractional programming problem, it is impossible to solve the problem directly. After substituting all given values, first change the probabilistic programming problem into deterministic equivalent programming problem using (5.6)-(5.7). In the next step, we transform the multi-objective fractional programming problem into equivalent multi-objective programming problem by the transformation given in (7.1)-(7.2) using $y_{n s t}=t x_{n s t}$. Finally, we apply $\epsilon$-constraint method to solve the multi-objective programming problem. Solving each objective functions separately subject to the given constraints, we obtain ideal solutions. By contracting pay-off matrix using each individual solution, we obtain the bounds of $\epsilon_{1}$ and $\epsilon_{2}$ which are in the bounds of the first objective function and second objective function respectively. $0.2657 \leq \epsilon_{1} \leq 0.456851$ and $0.001 \leq \epsilon_{2} \leq 0.005256$. By taking $\epsilon_{q}(q=1,2)$, we formulate the following two different single objective programming problem by taking one objective function as constraint. The first single objective programming problem is given by considering the second objective function as constraint.

$$
\min : Z_{1}=\sum_{n=1}^{2} \sum_{s=1}^{2} \sum_{t=1}^{7} o_{n} h_{s t} y_{n s t}
$$

subject to

$$
\begin{aligned}
& \sum_{s=1}^{2} \sum_{t=1}^{7} y_{1 s t} \geq \epsilon_{2}, \quad \sum_{n=1}^{2} \sum_{s=1}^{2} \sum_{t=1}^{7} r_{n} y_{n s t}=1, \quad \sum_{s=1}^{2} \sum_{t=1}^{7} y_{2 s t}=1, \\
& \sum_{n=1}^{2} h_{s t} y_{n s t} \leq t\left(\delta+\gamma \tan \left(\pi / 2-\eta_{s t} \pi\right)\right), \quad y_{n s t} \geq 0, \quad t>0 .
\end{aligned}
$$

Solving $(8.5)-(8.6)$ by taking different values of $\epsilon_{2}$ in $[0.001,0.005256]$ by LINGO software and applying the transformation $x_{n s t}=y_{n s t} / t, n=1,2$, 
Table 8. Solutions of $Z_{1}$ and $Z_{2}$ for different values of $\epsilon_{2}$.

\begin{tabular}{|c|c|c|c|c|c|}
\hline$\epsilon_{2}$ & 0.001 & 0.002 & 0.003 & 0.004 & 0.456 \\
\hline$x_{111}$ & 10 & 10 & 17 & 17 & 17 \\
\hline$x_{112}$ & 10 & 17 & 18 & 18 & 18 \\
\hline$x_{113}$ & 10 & 41 & 67 & 167 & 292 \\
\hline$x_{114}$ & 10 & 10 & 22 & 22 & 22 \\
\hline$x_{115}$ & 10 & 10 & 14 & 14 & 14 \\
\hline$x_{116}$ & 10 & 10 & 12 & 12 & 12 \\
\hline$x_{117}$ & 10 & 10 & 16 & 16 & 16 \\
\hline$x_{121}$ & 10 & 10 & 12 & 12 & 12 \\
\hline$x_{122}$ & 10 & 10 & 22 & 22 & 22 \\
\hline$x_{123}$ & 10 & 31 & 32 & 32 & 32 \\
\hline$x_{124}$ & 10 & 10 & 23 & 23 & 23 \\
\hline$x_{125}$ & 10 & 10 & 24 & 24 & 24 \\
\hline$x_{126}$ & 10 & 10 & 10 & 10 & 10 \\
\hline$x_{127}$ & 10 & 10 & 10 & 10 & 10 \\
\hline$x_{211}$ & 8 & 8 & 1 & 1 & 1 \\
\hline$x_{212}$ & 9 & 2 & 1 & 1 & 1 \\
\hline$x_{213}$ & 32 & 1 & 1 & 1 & 1 \\
\hline$x_{214}$ & 13 & 13 & 1 & 1 & 1 \\
\hline$x_{215}$ & 5 & 5 & 1 & 1 & 1 \\
\hline$x_{216}$ & 3 & 3 & 1 & 1 & 1 \\
\hline$x_{217}$ & 7 & 7 & 1 & 1 & 1 \\
\hline$x_{221}$ & 3 & 3 & 1 & 1 & 1 \\
\hline$x_{222}$ & 13 & 13 & 1 & 1 & 1 \\
\hline$x_{223}$ & 22 & 13 & 1 & 1 & 1 \\
\hline$x_{224}$ & 13 & 14 & 1 & 1 & 1 \\
\hline$x_{225}$ & 15 & 15 & 1 & 1 & 1 \\
\hline$x_{226}$ & 1 & 1 & 1 & 1 & 1 \\
\hline$x_{227}$ & 10 & 10 & 1 & 1 & 10 \\
\hline$Z_{1}$ & 0.2657 & 0.278395 & 0.321491 & 0.381491 & 0.4568510 \\
\hline$Z_{2}$ & 0.0014 & 0.002 & 0.0032 & 0.004 & 0.005256 \\
\hline
\end{tabular}

$s=1,2$ and $t=1,2, \ldots, 7$, the solution of the nurses scheduling programming problem is given by the Table 8 . The results in Table 8 shows that for different values of $\epsilon_{2}$, we obtain the compromise solution of the nurse scheduling problem which minimize the ratio of over time cost to regular time cost and maximize the ratio of number of senior nurses to number of junior nurses. If we take $\epsilon_{2} \geq 0.0032$, the number of junior nurses is much more less than number of senior nurses. The nurses manager has a great chance to select how many nurses must be assigned for each shift so as to a chive his objective.

The second single objective programming problem is maximized by considering the first objective function as constraint.

$$
\max : Z_{2}=\sum_{s=1}^{2} \sum_{t=1}^{7} y_{1 s t}
$$

subject to

$$
\sum_{n=1}^{2} \sum_{s=1}^{2} \sum_{t=1}^{7} o_{n} h_{s t} y_{n s t} \leq \epsilon_{1}, \quad \sum_{n=1}^{2} \sum_{s=1}^{2} \sum_{t=1}^{7} r_{n} y_{n s t}=1
$$




$$
\begin{aligned}
& \sum_{s=1}^{2} \sum_{t=1}^{7} y_{2 s t}=1, \quad \sum_{n=1}^{2} h_{s t} y_{n s t} \leq t\left(\delta+\gamma \tan \left(\pi / 2-\eta_{s t} \pi\right)\right), \\
& y_{n s t} \geq 0, \quad t>0 .
\end{aligned}
$$

Again solving (8.7)-(8.8) by taking different values of $\epsilon_{1}$ in the interval $[0.2657$, 0.4568510 ] by LINGO software and applying the transformation $x_{n s t}=y_{n s t} / t$, $n=1,2, s=1,2$ and $t=1,2, \ldots, 7$, the solution of the nurses scheduling programming problem is given by the Table 9 .

\begin{tabular}{|c|c|c|c|c|}
\hline$\epsilon_{1}$ & 0.26752 & 0.34 & 0.4 & 0.45685 \\
\hline$x_{111}$ & 10 & 17 & 17 & 17 \\
\hline$x_{112}$ & 10 & 18 & 18 & 18 \\
\hline$x_{113}$ & 10 & 98 & 198 & 292 \\
\hline$x_{114}$ & 10 & 22 & 22 & 22 \\
\hline$x_{115}$ & 10 & 14 & 14 & 14 \\
\hline$x_{116}$ & 10 & 12 & 12 & 13 \\
\hline$x_{117}$ & 10 & 16 & 16 & 16 \\
\hline$x_{121}$ & 10 & 12 & 12 & 13 \\
\hline$x_{122}$ & 10 & 22 & 22 & 2 \\
\hline$x_{123}$ & 10 & 32 & 32 & 32 \\
\hline$x_{124}$ & 10 & 23 & 23 & 23 \\
\hline$x_{125}$ & 10 & 24 & 24 & 24 \\
\hline$x_{126}$ & 10 & 10 & 10 & 10 \\
\hline$x_{127}$ & 10 & 10 & 10 & 10 \\
\hline$x_{211}$ & 8 & 1 & 1 & 1 \\
\hline$x_{212}$ & 9 & 1 & 1 & 1 \\
\hline$x_{213}$ & 32 & 1 & 1 & 1 \\
\hline$x_{214}$ & 13 & 1 & 1 & 1 \\
\hline$x_{215}$ & 5 & 1 & 1 & 1 \\
\hline$x_{216}$ & 3 & 1 & 1 & 1 \\
\hline$x_{217}$ & 7 & 1 & 1 & 1 \\
\hline$x_{221}$ & 3 & 1 & 1 & 1 \\
\hline$x_{222}$ & 13 & 1 & 1 & 1 \\
\hline$x_{223}$ & 22 & 1 & 1 & 1 \\
\hline$x_{224}$ & 14 & 1 & 1 & 1 \\
\hline$x_{225}$ & 15 & 1 & 1 & 1 \\
\hline$x_{226}$ & 1 & 1 & 1 & 1 \\
\hline$x_{227}$ & 10 & 10 & 10 & 10 \\
\hline$Z_{1}$ & 0.26572 & 0.345 & 0.42 & 0.456851 \\
\hline$Z_{2}$ & 0.0014 & 0.00331 & 0.00431 & 0.005256 \\
\hline
\end{tabular}

Table 9. Solutions of $Z_{1}$ and $Z_{2}$ for different values of $\epsilon_{1}$.

The results in Table 9 shows that for different values of $\epsilon_{1}$, we obtain the compromise solutions of the nurses scheduling problem which minimizes the ratio of over time cost to regular time cost and maximizes the ratio of number of senior nurses to number of junior nurses. If we take $\epsilon_{1}>0.26752$, the number of junior nurses is much more less than number of senior nurses.

From the two tables we see that a single point cannot optimize the two objective functions simultaneously. By choosing different values of $\epsilon_{1}$ in [0.2657, $0.4568510]$ and $\epsilon_{2}$ in $[0.001,0.005256]$, we obtain the compromise solutions of the nurse scheduling problem. 


\section{Conclusions}

In this study, we have considered MOPLFP problem, where the parameters of the right hand side constraints are random variables following Cauchy distribution. We have assumed other parameters of the mathematical model as deterministic variables. In the methodology, MOPLFP problem is converted to deterministic equivalent multi-objective linear fractional mathematical programming and the deterministic multi-objective linear fractional mathematical programming is transformed to multi-objective linear mathematical programming problem using the transformation $y_{j}=t x_{j}, t>0, j=1,2, \ldots, n$. The resulting multi-objective linear programming problem is solved using $\epsilon$-constraint method. Numerical example and a real life application are provided. Depending on different situations independent random variables following other distributions may be considered. This problem may be solved by using genetic algorithm approach.

\section{References}

[1] H.M. Babul and A. Sumi. Solving LFP by converting it into a single LP. International Journal of Operations Research, 8(3):1-14, 2011.

[2] M. Borza, A.S. Rambely and M. Saraj. Solving linear fractional programming problems with interval coefficients in the objective function. a new approach. Applied Mathematical Sciences, 6(69-72):3443-3459, 2012.

[3] M. Chakraborty and S. Gupta. Fuzzy mathematical programming for multi objective linear fractional programming problem. Fuzzy sets and systems, 125(3):335-342, 2002. https://doi.org/10.1016/S0165-0114(01)00060-4.

[4] V. Charles and D. Dutta. Linear stochastic fractional programming with branchand-bound technique. In Proceedings of the National Conference on Mathematical and Computational Methods, pp. 131-139, 2001.

[5] V. Charles and D. Dutta. Bi-weighted multi-objective stochastic fractional programming problem with mixed constraints. In Proceedings of the 2nd National Conference on Mathematical and Computational Methods, pp. 29-36. Allied New Delhi, India, 2003.

[6] V. Charles and D. Dutta. Linear stochastic fractional programming with sumof-probabilistic-fractional objective. Optimization Online, 2005.

[7] V. Charles and D. Dutta. Extremization of multi-objective stochastic fractional programming problem. Annals of Operations Research, 143(1):297-304, 2006. https://doi.org/10.1007/s10479-006-7389-7.

[8] V. Charles and D. Dutta. Identification of redundant objective functions in multi-objective stochastic fractional programming problems. Asia-Pacific Journal of Operational Research, 23(02):155-170, 2006. https://doi.org/10.1142/S0217595906000863.

[9] A. Charnes and W.W. Cooper. Programming with linear fractional functionals. Naval research logistics (NRL), 9(3-4):181-186, 1962.

[10] R. Enkhbat, Ya. Bazarsad and J. Enkhbayar. Convex-concave fractional minimization problem. J. Mong. Math. Soc, 15:3-10, 2011. 
[11] N. Güzel and M. Sivri. Taylor series solution of multi-objective linear fractional programming problem. Trakya Univ J Sci, 6(2):80-87, 2005.

[12] J.S. Kornbluth and R.E. Steuer. Multiple objective linear fractional programming. Management Science, 27(9):1024-1039, 1981. https://doi.org/10.1287/mnsc.27.9.1024.

[13] E. Marchi. Some remarks about the transformation of charnes and cooper. Tersedia di http://www. optimizationonline. org/DB FILE/2006/04/1381. pdf [9 Juli 2008], 2006.

[14] B. Martos and V. Whinston. Hyperbolic programming. Naval Research Logistics (NRL), 11(2):135-155, 1964. https://doi.org/10.1002/nav.3800110204.

[15] A.O. Odior. An approach for solving linear fractional programming problems. International Journal of Engineering \& Technology, 1(4):298-304, 2012.

[16] P. Ponnaiah and J. Mohan. On solving linear fractional programming problems. Modern Applied Science, 7(6):90, 2013.

[17] C.F. Ren, P. Guo, M. Li and J.J. Gu. Optimization of industrial structure considering the uncertainty of water resources. Water resources management, 27(11):3885-3898, 2013. https://doi.org/10.1007/s11269-013-0385-1.

[18] C.F. Ren, R.H. Li, L.D. Zhang and P. Guo. Multiobjective stochastic fractional goal programming model for water resources optimal allocation among industries. Journal of Water Resources Planning and Management, 142(10):04016036, 2016. https://doi.org/10.1061/(ASCE)WR.1943-5452.0000681.

[19] D. Roy. Fractional programming through genetic algorithm. In Computer, Communication, Control and Information Technology (C3IT), 2015 Third International Conference on, pp. 1-6. IEEE, 2015. https://doi.org/10.1109/C3IT.2015.7060175.

[20] S. Saha, M. Hossain, M. Uddin and R. Mondal. A new approach of solving linear fractional programming problem (LFP) by using computer algorithm. Open Journal of Optimization, 4(03):74, 2015. https://doi.org/10.4236/ojop.2015.43010.

[21] S. Schaible. Fractional programming. i, duality. Management science, 22(8):858867, 1976. https://doi.org/10.1287/mnsc.22.8.858.

[22] I.M. Stancu-Minasian. A fifth bibliography of fractional programming. Optimization, 45(1-4):343-367, 1999. https://doi.org/10.1080/02331939908844438.

[23] I.M. Stancu-Minasian. A sixth bibliography of fractional programming. Optimization, 55(4):405-428, 2006. https://doi.org/10.1080/02331930600819613.

[24] S.F. Tantawy. A new procedure for solving linear fractional programming problems. Mathematical and Computer Modelling, 48(5-6):969-973, 2008. https://doi.org/10.1016/j.mcm.2007.12.007.

[25] A. Udhayakumar, V. Charles and V.R. Uthariaraj. Stochastic simulationbased genetic algorithm for chance constrained fractional programming problem. International Journal of Operational Research, 9(1):23-38, 2010. https://doi.org/10.1504/IJOR.2010.034359.

[26] E. Valipour, MA. Yaghoobi and M. Mashinchi. An iterative approach to solve multiobjective linear fractional programming problems. Applied Mathematical Modelling, 38(1):38-49, 2014. 
[27] C.-F. Wen and H.-C. Wu. Using the parametric approach to solve the continuoustime linear fractional max-min problems. Journal of Global Optimization, 54(1):129-153, 2012. https://doi.org/10.1007/s10898-011-9751-9.

[28] H. Zhu and G.H. Huang. SLFP: A stochastic linear fractional programming approach for sustainable waste management. Waste Management, 31(12):26122619, 2011. https://doi.org/10.1016/j.wasman.2011.08.009.

[29] H. Zhu and G.H. Huang. Dynamic stochastic fractional programming for sustainable management of electric power systems. International Journal of Electrical Power \& Energy Systems, 53:553-563, 2013. https://doi.org/10.1016/10.1016/j.ijepes.2013.05.022. 